\title{
Not All Lies Are Spontaneous: An Examination of Deception Across Different Modes of Communication
}

\author{
Monica T. Whitty \\ Department of Media and Communication, University of Leicester, LE1 7RH, UK. E-mail: mw229@le.ac.uk \\ Tom Buchanan \\ School of Social Sciences, Humanities and Languages, University of Westminster, 309 Regent Street, \\ London W1B 2UW, UK
}

Adam N. Joinson

School of Management, University of Bath, Bath, BA2 7AY, UK

Alex Meredith
School of Social Sciences, Nottingham Trent University, Burton Street, Nottingham, NG1 4BU, UK

\begin{abstract}
An online diary study was performed to investigate deception across different media. One hundred and four individuals participated in the study, with 76 completing the diaries. Individuals were most likely to lie on the telephone. Planned lies, which participants also rated the most serious, were more likely told via SMS (short message service) text messaging. Most lies were told to people participants felt closest to. The featurebased model provides a better account of the deceptions reported by participants than do media richness theory or social distance theory. However, the authors propose a reworked feature-based model to explain deception across different media. They suggest that instant messaging should be treated as a near synchronous mode of communication. We suggest that the model needs to distinguish between spontaneous and planned lies.
\end{abstract}

In recent years, researchers have been especially interested in which information sources and services are deemed trustworthy and credible by their users (e.g., Hilligoss \& Rieh, 2008; Kelton, Fleischman, \& Wallace, 2008; Rieh, 2002). Researchers have found that individuals do hold concerns about digital sources of information, such as social networking sites, websites, and e-mail. Examples of such concerns include trust in virtual communities (Donath, 1998), e-commerce (Egger, 2000), and health information (Sillence, Briggs, Fishwick, \& Harris, 2004). Rieh (2002) theorizes that individuals are concerned with the quality of the information

Received February 18, 2011; revised August 2, 2011; accepted August 2, 2011

(C) 2011 ASIS\&T • Published online 29 September 2011 in Wiley Online Library (wileyonlinelibrary.com). DOI: 10.1002/asi.21648 found on the web and the authority of the source. All this uncertainty regarding the Internet as a trustworthy space has led scholars to question whether people are more likely to be deceptive online than in more traditional spaces, such as face-to-face and the telephone. Given the uncertainly regarding trustworthiness of information conveyed through digital media, in this study we were interested in the prevalence of deception across different media. Our study was interested in the sorts of communication that transpires between individuals rather than focusing, as some previous researchers have, on one-way communications (e.g., websites).

Lying is, of course, a part of our everyday lives, with people telling an average of one to two lies per day (DePaulo, Kashy, Kirkendol, Wyer, \& Epstein, 1996). In the past, researchers have focused on the motivations for lying (Camden, Motley, \& Wilson, 1984), the types of lies people tell (DePaulo et al., 1996), frequency of lying (DePaulo et al., 1996), and whom individuals tell their lies to (Williams, 2001). More recently, researchers have focused on the medium in which the lie is told (Hancock, Thom-Santelli, \& Ritchie, 2004; Whitty \& Carville, 2008), and the linguistic analysis of mediated deception to identify cues to deceptive communications (Hancock, Curry, Goorha, \& Woodworth, 2008; Zhou, Burgoon, Nunamaker, \& Twitchell, 2004). The study of deceptive practices across different media is important because of what it can tell us about the nature of deception. For instance, Hancock et al. (2004) found that the prevalence of deceptive communications is greatest in synchronous, nonrecorded media (e.g., the telephone), and least prevalent in recorded, asynchronous media (e.g., e-mail). This suggests, first, that the majority of lies tend to occur 
in an unplanned, spontaneous manner, and second, that when engaged in deceptive communication, people pay as much attention to the likelihood of a record being kept as to the absence of visual or verbal cues.

In the present study, we are concerned with four key issues. The first is the prevalence of deceptive communications in different media. Earlier research (e.g., Hancock et al., 2004) using diary studies suggested that deception is more prevalent in face-to-face and telephone settings than in mediated communication. However, the sample used in the Hancock study was small $(n=28)$, which when combined with the low occurrence of deception, made cross-media comparisons impossible. In the present study, we address this shortcoming by recruiting more participants while utilizing the same diary methodology. The second issue we address is whether lies tend to be planned or spontaneous, and how that varies by media and the seriousness of the lie. As noted above, most lies tend to be spontaneous, and as such they tend to occur in spontaneous interactions, whereas planned lies may occur more frequently in asynchronous media (Hancock et al., 2004). In the present study, we examine not only the nature of the lie (i.e., spontaneous vs. planned) across media, but also the seriousness of the lie, and how that relates to its nature. The third issue we address is whether the lie is focused on enhancing or protecting the self, or the target of the lie, and how that differs across media. The final issue we address is the relationship between the deceiver and the target, and how that relates to the media used to lie. In the following sections, we review the existing literature on each of these issues in turn, and present hypotheses based on this review.

\section{Lying in Different Modes of Communication}

Theories that have been devised to account for which mode of communication individuals are more likely to lie in have focused on the social distance between the liar and the target, the richness of the media, and some of the specific features of the media. Three of the main theories are social distance theory, media richness theory, and the feature-based model.

Social distance theory suggests that because lying makes individuals feel uncomfortable, they will choose less-rich media to maintain social distance between themselves and the person they are lying to (DePaulo et al., 1996); that is, they will avoid media which transmit cues that people believe will give away deceit (e.g., voice, body language). Moreover, in less-rich media the deceiver has more control over the interaction - any unexpected questions can be thought about, rather than being responded to immediately. This theory would predict that individuals are more likely to lie in e-mail and SMS (short message service; i.e., text messaging), followed by instant messaging, then social networking sites, then telephone, and lastly face-to-face.

In contrast, media richness theory argues that because lying is highly equivocal, individuals elect to lie more in rich media, which includes multiple cue systems, immediate feedback, natural language, and message personalization
(Daft \& Lengel, 1986; Trevino, Lengel, \& Daft, 1987). This theory would predict that individuals are more likely to lie in face-to-face situations, followed by telephone, then social networking sites, then instant messaging, and finally e-mail and text messages.

Finally, the feature-based model, as the name suggests, is more concerned with the different elements of particular media (see Table 1). Some media, for instance, allow communication to take place in real time (e.g., face-to-face, phone), whereas other media allow one to keep a record of the interaction to read back on (e.g., post, e-mail). This model purports that, when considering deception, there are three aspects of the media need to be taken into consideration: whether the media is synchronous, recordless, and distributed (i.e., not copresent) communication (Hancock et al., 2004). One of the strengths of this theory is that unlike early theorists, Hancock et al. do not assume the Internet is a homogenous space. Instead, digital technologies and the variety of spaces online where we seek information and communicate have their own unique qualities. The feature-based model proposes that lying is more likely to take place when the media is synchronous and distributed, but nonrecordable. One lies more in synchronous interactions because most lying is spontaneous; hence, synchronous communication should present more opportunities to lie. Distributed media allow one to feel less present; thus, lying is more comfortable. In recorded communication, one is aware that their conversation is potentially kept or stored (e.g., in a saved e-mail) and can be referred to in future conversations; hence, people are less likely to lie if they are aware that there is proof of their lie that can be referred to later. This theory would predict that people are more likely to lie on the telephone, followed by both face-to-face interactions and instant messaging, followed by social networking sites, e-mail, and SMS (text messages) (see Table 1).

In their diary study, Hancock and colleagues report that $37 \%$ of telephone-based interactions contained a lie, compared to $27 \%$ of face-to-face (FTF), $21 \%$ of instant messaging (IM), and $14 \%$ of e-mail. They interpret this as providing empirical support for the feature-based model of deception. However, their study was limited to a small sample size of 28 participants and collected lies in four modes: face-to-face, telephone, e-mail, and instant messaging. In the present study, we focus on six modes of communication: FTF, telephone, social networking sites (SNS), IM, e-mail, and text messaging (SMS). Based on Hancock et al.'s findings our first hypothesis is that more lies will be told by the telephone than FTF and IM, followed by SNS, e-mail, and SMS.

\section{Spontaneous Versus Planned Lies}

The feature-based model assumes that lying is spontaneous. Although most lies are spontaneous, there is also evidence that a fair proportion of lies are planned (DePaulo, Kashy, et al., 1996, 2003). For example, if an adolescent planned to engage in an unapproved activity and would arrive home late, would he or she decide to wait until returning home 
TABLE 1. Feature-Based Model (Adapted From Hancock et al., 2004), Ranking Predictions on Likelihood of Lying.

\begin{tabular}{lccccc}
\hline & FTF & Phone & IM & E-mail & SNS \\
\hline Media features & & & & & \\
Synchronous & $\mathrm{x}$ & $\mathrm{x}$ & $\mathrm{x}$ & $\mathrm{x}$ & \\
Recordless & $\mathrm{x}$ & $\mathrm{x}$ & $\mathrm{x}$ & $\mathrm{x}$ & 3 \\
Distributed & 2 & 1 & 2 & 3 & 3 \\
Lying predictions & & & & 3 \\
Feature-based & & & & & \\
\hline
\end{tabular}

Note FTF = Face-to-face; IM= instant messaging; SNS = social networking sites; SMS = text messaging (short message service).

to lie to a parent or send a text before arriving home (to pave the way so to speak)?

Some theorists have argued that lying is suggestive of game theory (Schelling, 1968). They have argued that choosing to lie or not lie can be strategic. To be strategic, however, one needs time to consider and potentially plan a lie. Given that communication theorists have argued that one can be more strategic when communicating in some online spaces (e.g., Walther, 1996), we might expect that planned lies are more commonly told in asynchronous forms of communication. Based on the above literature our second hypothesis is that planned lies will be more likely to occur in asynchronous media (e.g., e-mail, SMS, SNS) than in synchronous media (e.g., FTF, phone, IM). This proportion will differ across media.

\section{Serious Lies}

DePaulo, Ansfield, Kirkendol, and Boden (2004) have argued that "the little lies of everyday life are overwhelmingly lies that are not very serious" (p. 148). But what of the more serious lies? Although we acknowledge the philosophical debates concerning the seriousness of lies and when it might be permissible to lie, if at all, (see for example, Baillie, 2000; Kant, 1997) we were more concerned, as aligned with previous research (see DePaulo, 2004), with how participants rated their own lies. Evidence has found a positive relationship between planned lies and serious lies (DePaulo et al., 2004). Based on DePaulo et al.'s research, our fourth hypothesis is that planned lies are more likely to be serious than unplanned lies.

\section{Self-Oriented and Other-Oriented Lies}

DePaulo et al. (1996) have argued that "many of the lies of everyday life are told to avoid tension and conflict and to minimize hurt feelings and ill will" (p. 980). With this in mind, theorists have argued that when researching lies we need to be mindful of the type of lie being told (DePaulo, Ansfield, et al., 1996; Whitty \& Carville, 2008). Some lies are told to protect or enhance the liar psychologically (selforiented), whereas others are told to protect or enhance the target (other-oriented). DePaulo, Kashy, et al. (1996) contend that other-oriented lies are altruistic; however, not everyone would agree. Kaplar and Gordon (2004) argue that otheroriented lies are sometimes perceived as altruistic by the teller, but not by the receiver. Considerations of the receiver were beyond on the focus of this article, and we do not purport that other-oriented lies are necessarily altruistic. Instead, we were interested in the perceptions of the participant. In previous work, DePaulo et al. found that self-serving lies were more frequent than other-oriented lies. Based on DePaulo et al.'s study, our fifth hypothesis is that participants will tell more self- than other-directed lies.

Whitty and Carville (2008) investigated whether the feature-based model is still supported when considering these two types of lies. In their study, they presented participants with hypothetical scenarios of other-oriented and self-oriented lies and asked them to rate the likelihood of telling the lie. They found that the social distance model was better at predicting self-serving lies. This study is limited in that, because lying is typically spontaneous, it is important to consider any differences across media with "real life" data. With little theory or empirical evidence to theorize about any differences, our hypothesis for this research question is exploratory. Our sixth hypothesis is the proportion of selfand other-directed lies will differ across media.

\section{The Relationship With Target of the Lie}

Diary studies have revealed that individuals are less likely to lie to those they are in close relationships with (DePaulo \& Kashy, 1998). These results make theoretical sense because deception is often frowned upon and individuals typically want to maintain ties with those close to them. Moreover, individuals close to us know more information about us and so could potentially spot a lie more easily. It has also been found that when people do lie to people they feel close to these lies are usually other-oriented (DePaulo \& Kashy). Again, this is intuitive because other-oriented lies are told to protect the target of the lie.

Based on DePaulo and Kashy's (1998) research we arrived at two hypotheses. First, that participants will tell fewer lies to those they are compared to those they are not. Second, that there will be an interaction between social relationship and type of lie, such that most lies to close others will be other-oriented, whereas most lies to distant others will be self-oriented. 


\section{Method}

For this study we drew from the work of Hancock et al. (2004) and DePaulo et al. (1996). We devised a similar diary study, but added some further questions to reflect our additional research objectives. Moreover, we included additional modes of communication for participants to consider.

\section{Participants}

Participants were undergraduate students studying in the School of Social Sciences at a large university in the United Kingdom. Overall, 104 people participated in the study, with 76 participants completing their diaries in full. Analyses of people's lying behavior (e.g., comparison of number of lies told in each medium) are restricted to the 76 who provided complete data because the person is treated as the unit of analysis. However, analyses of the lies themselves (e.g., level of planning of lies in each medium) use the entire dataset, because the lie is treated as the unit of analysis. Ages ranged from 18 to 32 years, with a mean age of $19.45(S D=1.836)$. There were many more women $(68 ; 89 \%)$ in the sample than men $(8 ; 11 \%)$. Participants were offered the incentive of either a shopping voucher worth $£ 10$ or research credits for their final year projects. They were all also given a chance to win an Amazon voucher of $£ 300$ (these incentives have been used in similar studies on deception).

\section{Materials}

Before commencing the diary study, participants were required to complete a form providing demographic details about themselves as well as a series of psychological measures such as self-esteem and Internet self-efficacy (not reported here). The Social Interaction and Deception Form first designed by DePaulo et al. (1996) and later reworked by Hancock et al. (2004) was slightly modified for this study. We placed this form online, and our participants were required to complete the form for seven consecutive days.

In the social interaction section, individuals were asked to consider for that day approximately how many interactions they had in different media, the gender and number of interaction partners, and how well they knew the people they were communicating with. In the deception section, participants were required to report their lies (no matter how trivial, including white lies), stating the content, the reason for the lie, the type of lie, and the referent of the lie (how close they were to the referent and his or her gender). They were asked to say in which media the lie took place as well as approximately how many people they told the lie to at the same time. They were asked to rate the purpose of the lie, the type of lie, and how many times they had previously told this lie. On Likert scales they were asked to report how planned ("How planned was the lie?"; rated from Completely Spontaneous to Carefully Planned), serious ("How serious was the lie?"; rated from Trivial to Very Serious), and believable ("How believable do you think the lie was?"; rated from Very Unbelievable to Very Believable $)(1=$ Least, $9=$ Most $)$. They were also asked to rate how comfortable they felt during the lie and after the lie, how the target would have felt if told the truth, how they would have felt had they told the truth, and how important it was to not get caught out $(1=$ Least, $9=$ Most $)$. Here we focus on the answers to a subset of these questions.

Participants were also given pocket notebooks to record brief details of their lies throughout the day as an aide memoire before completing the form. Participants received daily reminders via e-mail and SMS to prompt submission of the form. Moreover, reminders were sent the next day to any participants who had not submitted the previous day's forms.

\section{Procedure}

The study received ethical clearance from the host university. The names of participants remained separate to their forms (where they were given an ID number) so that they could remain anonymous and participants were able to withdraw from the study at any point if they so desired.

Prior to the main study, a pilot test was run to ensure that participants understood the instructions and to see how difficult it would be for participants to complete a 7-day diary. The pilot test suggested that more reminders were necessary and so we included SMS reminders in the main study.

Participants initially attended a one-hour group session where the purpose of the research and what was expected of them were laid out. They were given paper copies of the instructions, which were also available when they logged in to complete their diaries. As with DePaulo et al.'s (1996) and Hancock et al.'s (2004) studies, participants were told that the researchers did not condone or condemn lying, but instead were examining lies scientifically to explore lying behavior. DePaulo et al.'s definition of a lie was given: "A lie occurs any time you intentionally try to mislead someone" (p. 981). They were also told to record all lies, big and small. In addition, they were told that lies included all attempts to mislead, including nonverbal lies. The way we have operationalized a lie is consistent with previous research (e.g., DePaul et al., 1996; Hancock et al., 2004).

The online forms needed to be completed each day and the research assistant checked each morning the next day to see if everyone had filled them in. If not, they were sent an additional reminder. Participants were also encouraged to write notes each day to assist their recall.

\section{Results}

Participants who submitted data for all days of the study on average reported $149.68(S D=59.09)$ interactions across all the media. The smallest number of communications recorded was 42 and the highest was 333. The number of lies participants reported telling ranged from 0 to 18 , with a mean of 6.25 $(S D=3.65)$ over the entire week. On average, the percentage of interactions that were deceptive was $4.91 \%(S D=3.82)$, with the percentage ranging from 0 to $22.81 \%$.

H1: More lies will be told by the telephone than FTF and IM, followed by SNS, e-mail and SMS. 
TABLE 2. Communications and Lies Reported by Each Person Across Entire Week.

\begin{tabular}{lccr}
\hline Medium & $\begin{array}{c}\text { Mean number of } \\
\text { communications }(S D)\end{array}$ & $\begin{array}{c}\text { Mean number of } \\
\text { lies }(S D)\end{array}$ & $\begin{array}{c}\text { Mean lie index } \\
(S D)\end{array}$ \\
\hline Face-to-face & $49.64(21.69)$ & $3.46(2.32)$ & $0.08(0.06)$ \\
Phone & $13.17(9.19)$ & $1.46(2.52)$ & $0.13(0.17)$ \\
E-mail & $5.25(5.07)$ & $0.11(0.31)$ & $0.03(0.14)$ \\
Instant messaging & $12.55(16.03)$ & $0.37(0.63)$ & $0.04(0.07)$ \\
Social network site & $11.13(10.55)$ & $0.08(0.27)$ & $0.01(0.07)$ \\
SMS (text message) & $57.58(37.17)$ & $0.72(1.07)$ & $0.02(0.04)$ \\
Other medium & $0.36(1.06)$ & $0.07(0.30)$ & $0.13(0.29)$ \\
\hline
\end{tabular}

To evaluate the relative frequency of lies across media, the level of use of each medium needs to be taken into account: People might appear to lie more in FTF settings than by e-mail, simply because they have more FTF interactions. Accordingly, a "lie index" was created for each medium: The number of lies each participant told in a specific medium was divided by the total number of interactions he or she reported having in that medium. The lie index shown in Table 2 thus represents the proportion of interactions in a medium that were lies over the entire week.

To control for individual differences in honesty and patterns of media use, each of the lie index variables was then treated as one observation in a one-way repeated-measures ANOVA. The assumption of sphericity was violated, so multivariate tests were used to assess effects. There was a significant effect for medium, Pillai's Trace $=0.653, F$ $(5,39)=14.654, p<0.0005$. Lies were told significantly more often in some media than others.

Post hoc comparisons (least significant difference tests) indicated that people are most likely to lie by telephone (13\% of interactions involved deception), with FTF coming in second ( $8 \%$ of interactions involved deception). Phone calls are significantly $(p=0.044)$ more likely to involve deception than face-to-face interactions. Both are significantly ( $p<0.005$ for all comparisons) more likely to be used for lying than the other media, where there are no significant differences other than people being less likely to lie by SMS than by IM ( $p=0.042)$. Thus, $\mathrm{H} 1$ is only partially supported. However, as Table 2 indicates, very few lies were actually told using e-mail, IM, or SNS, which makes interpretation difficult.

$\mathrm{H} 2$ : Planned lies will be more likely to occur in asynchronous media (e.g., e-mail, SMS, SNS) than in synchronous media (e.g., FTF, phone, IM). This proportion will differ across media.

To test this hypothesis, level of planning for lies was compared across media using a one-way ANOVA. The media examined were restricted to FTF (329 lies), telephone (147 lies), and SMS (68 lies) due to the low number of lies in other media. There was a significant effect of medium, $F$ $(2,541)=5.11, p=0.006$. Post hoc tests (Tukey HSD) indicated that FTF $(M=3.50, S D=2.30)$ and SMS $(M=4.44$, $S D=2.11$, differed significantly $(p=0.007)$. Telephone, which had a mean score between the two $(M=3.90$, $S D=2.47$ ) did not differ significantly from either. This result is partially consistent with the hypothesis that lies told in the asynchronous medium (SMS) had a higher level of planning than one of the synchronous media (FTF). It is worth noting that the levels of planning were relatively low in all media (planning was rated on a 1-9 scale anchored at Completely Spontaneous and Carefully Planned).

\section{H3: Planned lies are more likely to be serious than unplanned} lies.

The hypothesized link between level of planning and perceived seriousness was observed. For the entire sample of lies, perceived seriousness of lie and level of planning were significantly correlated $(r=0.317, n=598, p<0.0005)$. Lies that were planned more carefully tended to be rated as more serious.

H4: Participants will tell more self- than other-directed lies.

Participants were asked to indicate the beneficiary of each lie: whether it was intended to protect or benefit themselves, the target, neither, or both. For each participant, the total number of lies of each type told over the week was treated as one observation in a one-way repeated-measures ANOVA. The assumption of sphericity was violated, so multivariate tests were used to assess effects. There was a significant effect for medium, Pillai's Trace $=0.511, F(3,73)=25.42$, $p<0.0005$. Post hoc pairwise comparisons (least significant difference statistic) indicated that significantly more lies were told to protect/benefit the self than any of the other motivations. None of the other motivations differed in the number of lies they inspired. This is consistent with the hypothesis that participants would tell more self-oriented lies.

H5: The proportion of self- and other-directed lies will differ across media.

Given the low number of lies in some media, the proportions of self- and other-directed lies were examined only for FTF, telephone, and SMS. A chi-square test of association between lie medium (FTF, telephone, SMS) and lie reason (self, other, both, neither) did not indicate a link, $\chi^{2}(6,543)=8.50, p=0.20$ : Types of lies were equally 


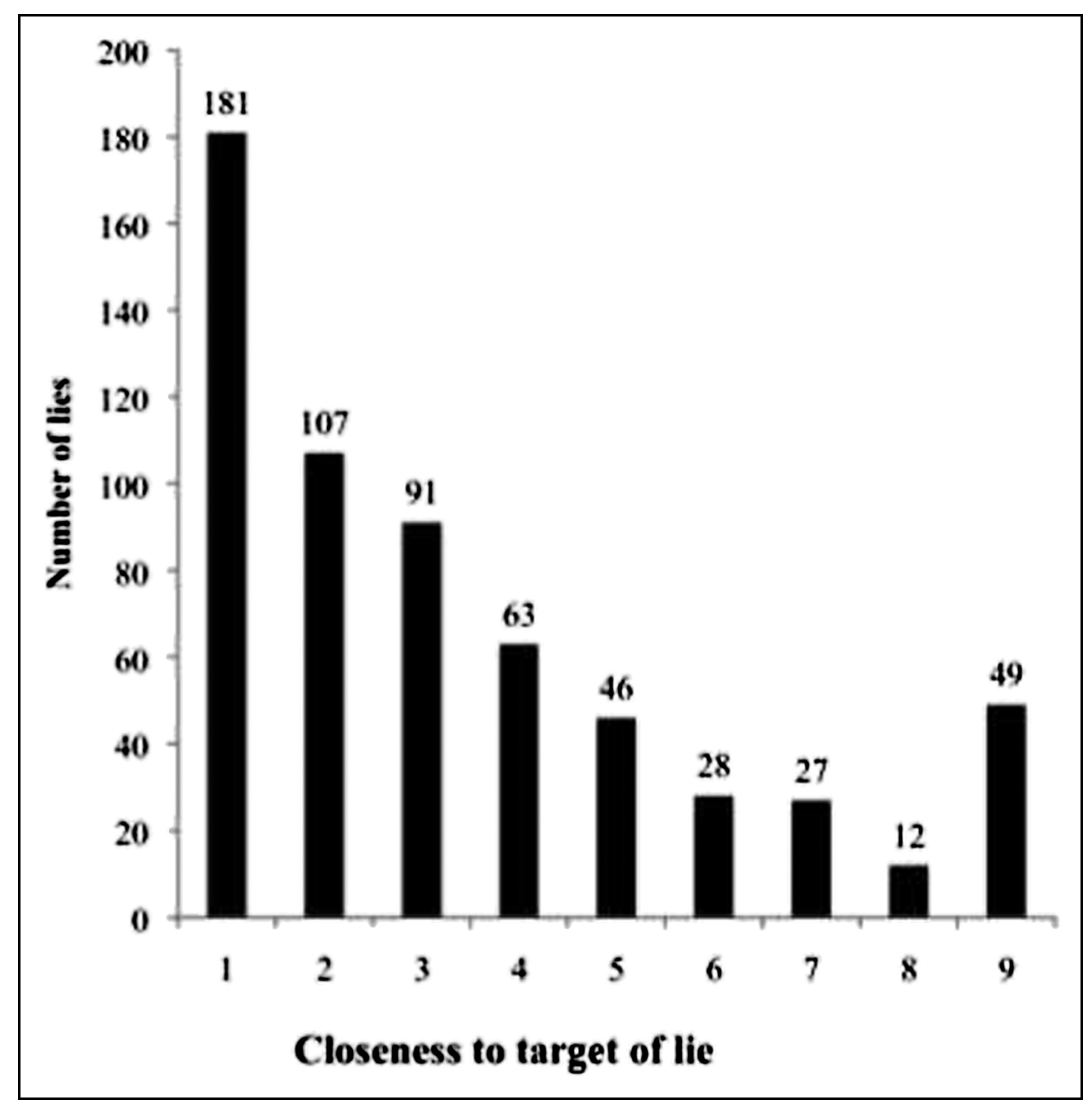

FIG. 1. Lies told to people of varying degrees of closeness.

distributed across the three media. Hypothesis 5 was therefore rejected.

H6a: Participants will tell fewer lies to those they are close to compared to those they are not close to.

Participants rated the targets of their lies on a 9-point scale (from Very Close to Complete Stranger) as to their degree of closeness. The hypothesis was tested by comparing the frequencies of lies told to targets in each of the nine levels of the closeness variable. A chi-square test for goodness of fit indicated that number of lies told was not distributed evenly across the different levels of closeness, $\chi^{2}(8,604)=329.29$, $p<0.0005$. Most lies were told to people very close to the liars, with decreasing numbers as closeness decreased until the final category-total strangers-where there is a spike. The pattern is shown in Figure 1.

H6b: There will be an interaction between social relationship and type of lie, such that most lies to close others will be other-oriented, whereas most lies to distant others will be self-oriented.

To test this hypothesis, the level of closeness variable was collapsed to three levels rather than the original nine (this was done to avoid small cell sizes). Analysis was restricted to lies that were purely self- or other-oriented: Lies intended to protect or benefit either both or neither were excluded.
A chi-square test for association indicated there was a link between motivation for the lie and closeness of the target, $\chi^{2}(2,414)=6.62, p=0.037$. Although most lies are selforiented, the proportion that are for the benefit of the target is lower in the least-close grouping ( $9 \%$ for benefit of target) than in the other two groups $(28 \%$ in the closest group, $31 \%$ in the middle group). This is consistent with the notion that lies told to distant others will be more likely to be self-oriented.

\section{Discussion}

The present study sought to extend upon previous work on deception across different modes of communication. Our intention was to first examine theories that have been set out to predict the medium people are most likely to lie in and then to see if these theories extend to all types of lies (i.e., planned vs. spontaneous; lies to benefit the self vs. lies to benefit others). Moreover, we were interested in whether the medium people are more likely to lie in varies according to the target of the lie (i.e., level of closeness).

Our first hypothesis, which predicted, according to Hancock et al.'s (2004) feature-based model, that lies will be told more via the telephone than FTF and IM, followed by SNS, e-mail, and SMS was only partly supported. This theory proposes that the more synchronous and distributed, but the less recordable a medium is, the more frequently lying will occur. 
To examine this we created a lie index so that the lies a person told over the entire week in each medium was divided by the total number of interactions they reported having in that medium. Akin to Hancock et al.'s (2004) research, we found that people were more likely to lie on the telephone followed by FTF. Moreover, as the feature-based model would predict, there were no differences between FTF communication and IM; however, we found that more people are likely to lie on the phone and FTF than any of the digital modes of communication. Hancock et al.'s model would have predicted significant differences between IM and the other digital modes of communication; however, our findings did not support this prediction. Instead, the only other significant finding was that SMS was used significantly less than IM for telling lies. Again, this supports the feature-based model. However, the model also predicts differences between IM and e-mail and IM and SNS, which our study did not find. This suggests that Hancock et al.'s model can account only in part for where people are more likely to lie. Perhaps they were unable to tease apart the differences we have in our study, because of the sample size of only 28 participants. However, we also note that our sample size was too small to examine in detail each of the digital media we included in our study. It is nonetheless noteworthy that the data also do not support the other two main theories, the social distance theory and media richness theory.

In reflection, we might want to reconsider the feature of synchronicity that Hancock et al. argue is a feature of IM. Contrary to those researchers' assertion, others have described IM as only "near synchronous" (see Nardi, Whittaker, \& Bradner, 2000). Perhaps that short time gap in responding counts. Given that lying is mostly spontaneous, when presented with an opportunity to reflect, individuals might opt to be honest. Instead, we might argue that one quality that all the digital technologies included in our study share is that they are asynchronous. Moreover, Hancock et al. have argued that there should be no significant differences between FTF and IM because they both have two of the features in the model. These features we note are not the same (FTF being synchronous and recordless and IM being synchronous and distributed); so it may well be that the features proposed in Hancock's model do not bear equal weighting. At present, we can confidently point out the feature-based model as it stands is not perfect and further research with even larger sample sizes is needed to test our claims. Moreover, future considerations of the feature-based model might reveal other elements.

In this study, we examined how online communication between individuals compared with more traditional spaces, such as face-to-face and telephone, in terms of how much deception occurred. Our results suggest that communication that takes place via some digital technologies is more trustworthy than other media. Researchers might want to open up their research to comparisons of trustworthiness of information sources across different media. We do recognize that a limitation of our study is that we have not examined all possible digital means of communication. In future studies, researchers might examine deliberate attempts to deceive via more one-way communications (e.g., websites).

Importantly, the presented study acknowledged that not all lies are spontaneous; so we questioned whether the featurebased model holds when considering planned lies. As with previous research, we found that planned lies were rarer than spontaneous lies. Due to the low number of lies in the other media, we were able to compare only FTF with telephone and SMS. Here we find the opposite result to the one above - that most planned lies were told via SMS and this significantly differed to FTF. This result is partly consistent with our hypothesis that given the level of planning involved a more asynchronous environment is a more ideal place to tell a planned lie. Moreover, SMS might be preferred to the other digital technologies (which due to low numbers we were not able to analyze) because it is the most lean medium (making it easier to write something more equivocal). Important to our understanding of this result is our finding that planned lies tend to be more serious lies.

Previous researchers have argued that when examining which mode of communication people are more likely to lie in, we should separate self-oriented (i.e., lies to protect or enhance the liar) and other-oriented lies (i.e., lies to protect or enhance other people psychologically; Whitty \& Carville, 2008). However, previous research has considered only hypothetical situations. Consistent with previous research (see DePaulo et al., 1996) we found that our sample was overall more likely to tell self-oriented lies. Given the lack of real-life data for this research question, we simply hypothesized that there would be a difference in the relative rates of self- and other-oriented lies across the different media. Again, the low number of lies in some of the media restricted our analysis to FTF, telephone, and SMS; and revealed no association between the orientation of the lie and the medium. This limitation to three media to examine makes it difficult to arrive at any firm conclusions, except to say that it appears that when considering lies across different media we do not need to consider the orientation of the lie.

In addition to the type of lie, we were interested in examining how lying might vary depending on the target of the lie. Do we lie more to people we are closer to? Do we tell more other-oriented lies to those we have a closer relationship with? Not surprisingly, we found that people tell more lies to those toward whom they have a feeling of closeness. Given that we did not ask participants to provide information about the target they engaged in each interaction with in the week, we were unable to determine whether this was because the participants had more opportunities to lie to those who were close to them (although we can fairly safely assume this to be the case). What is perhaps more interesting is that the number of lies rises again when it comes to lying to strangers. Perhaps this is because individuals anticipate they will never see them again. Based on previous research (see Whitty \& Carville, 2008), it seemed reasonable to hypothesize that most lies told to those individuals one is close to are other-oriented and those lies told to distant others are more self-oriented. To test this hypothesis, we collapsed levels of closeness (originally nine 
categories) into three levels. We found that though most lies are self-oriented the proportion told that were other-oriented were most likely told to those with whom one felt close. This distinction is important given that other-oriented lies serve more altruistic purposes than self-oriented lies. Finally, we wanted to examine whether self- and other-oriented lies to targets one felt close and least-close to varied across the different modes of communication. However, given the low expected counts we were unable to carry out this analysis.

\section{Limitations and Future Research}

The strength of a diary study is that participants are able to self-report their lying behavior, rather than participants guessing how they might act given a particular scenario. Comparing this study with other research findings highlights that we do obtain different results using these two types of methods.

Nonetheless, given the cumbersome nature of filling out a diary we were forced to restrict the amount of information gathered (e.g., more details about each interaction would be desirable). Future research might drop some of the variables we used in this study to ask more details about each interaction. Moreover, this study highlights the need to collect a larger sample. Finally, although we acknowledge the possibility that respondents might have been less inclined to report lies due to issues of social desirability, we do note that the average number of lies reported is similar to previous studies. In fact, the consistency with two other similar diary studies (DePaulo et al., 1996; Hancock et al., 2004) is striking. Participants were completely anonymous, and filled out their diaries online, which as previous studies demonstrate, limits the problems associated with participants responding in more socially desirable ways (Joinson, 1999).

The results from this study reveal more about which theoretical model best predicts lying across different modes of communication. The feature-based model still seems to be the preferred model over the social distance theory and media richness theory. However, we would suggest that the featurebased model needs to acknowledge IM as a near-synchronous rather than synchronous mode of communication, which then changes what this model would predict. Researchers might also consider adding lean media as a feature in this model. This, we argue, might explain why individuals tell more planned lies via SMS text messages than other media. The weighting of each of these features also needs to be considered in future studies. Moreover, as our study found, the feature-based model does need to distinguish between spontaneous and planned lies.

Although our sample size did not allow us to examine whether lies across media vary according to the type of lie (self-oriented vs. other-oriented) and target of the lie (level of closeness) we did find that our participants told more otheroriented lies to those they are close to. Future research might want to investigate if this does vary across the different media.

Digital technologies are constantly changing and developing. For example, accessing e-mail via one's mobile phone is increasing in popularity. We need to be mindful of how these technologies develop and how individuals appropriate them into their everyday lives. The media in which individuals are more likely to tell planned and spontaneous lies could well change as the features of these media change.

\section{Acknowledgment}

The work reported in this paper was funded by a British Academy grant.

\section{References}

Baillie, J. (2000). Hume: On morality. London: Routledge.

Camden, C., Motley, M.T., \& Wilson, A. (1984). White lies in interpersonal communication: A taxonomy and preliminary investigation of social motivations. Western Journal of Speech Communication, 48, 309-325.

Daft, R., \& Lengel, R. (1986). Organizational information requirements, media richness and structural design. Management Science, 32(5), 554-571.

DePaulo, B.M. (2004). The many faces of lies. In A.G. Miller (Ed.), The social psychology of good and evil (pp. 303-326). New York: Guilford Press.

DePaulo, B.M., Ansfield, M.E., \& Bell, K.L. (1996). Theories about deception and paradigms for studying it: A critical appraisal of Buller and Burgoon's interpersonal deception theory and research. Communication Theory, 3, 297-310.

DePaulo, B.M., Ansfield, M.E., Kirkendol, S.E., \& Boden, J.M. (2004). Serious lies. Basic and applied social psychology, 26 (2\&3), 147-167.

DePaulo, B.M., \& Kashy, D.A. (1998). Everyday lies in close and casual relationships. Journal of Personality and Social Psychology, 74, 63-79.

DePaulo, B.M., Kashy, D., Kirkendol, S.E., Wyer, M.M., \& Epstein, J.A. (1996). Lying in everyday life. Journal of Personality and Social Psychology, 70(5), 979-995.

DePaulo, B.M., Lindsa, J.J., Malone, B.E., Muhlenbruck, L., Charlton, K., \& Cooper, H. (2003). Cues to deception. Psychological Bulletin, 129(1), 74-118.

Donath, J.S. (1998). Identity and deception in the virtual community. In M.A. Smith \& P. Kollock (Eds.), Communities in cyberspace (pp. 29-59). New York: Routledge.

Egger, F.N. (2000). Trust me, I'm an online vendor: Towards a model of trust for e-commerce system design. In Proceedings of the Conference on Human Factors in Computing Systems (pp. 101-102). New York: ACM Press.

Kant, I. (1997). Groundwork of the metaphysics of morals. In M. Gregor (Ed. and Trans.). Cambridge: Cambridge University Press. (Original work published in 1800)

Kaplar, M.E., \& Gordon, A.K. (2004). The enigma of altruistic lying: Perspective differences in what motivates and justifies telling within romantic relationships. Personal Relationships, 11(4), 489-507.

Kelton, K., Fleischmann, K.R., \& Wallace, W.A. (2008). Trust in digital information. Journal of the American Society for Information Science and Technology, 59(3), 363-374.

Hancock, J., Thom-Santelli, J., \& Ritchie, T. (2004). Deception and design: The impact of communication technologies on lying behavior. In Proceedings of the Conference on Computer Human Interaction (pp. 130-136). New York: ACM Press.

Hancock, J.T., Curry, L., Goorha, S., \& Woodworth, M.T. (2008). On lying and being lied to: A linguistic analysis of deception. Discourse Processes, $45,1-23$.

Hilligoss, B., \& Rieh, S.Y. (2008). Developing a unifying framework of credibility assessment: Construct, heuristics, and interaction in context. Information Processing and Management, 44, 1467-1484.

Joinson, A. (1999). Social desirability, anonymity, and Internet-based questionnaires. Behavior Research Methods, Instruments and Computers, 31(3), 433-438.

Nardi, B.A., Whittaker, S., \& Bradner, E. (2000). Interaction and outreaction: Instant messaging in action. In Proceedings of the 2000 ACM Conference 
on Computer Supported Cooperative Work (pp. 79-88). New York: ACM Press

Rieh, S.Y. (2002). Judgement of information quality and cognitive authority in the web. Journal of the American Society for Information Science and Technology, 53(2), 145-161.

Schelling, T.C. (1968). Game theory and the study of ethical systems. The Journal of Conflict Resolution, 12(1), 34-44.

Sillence, E., Briggs, P., Fishwick, L., \& Harris, P. (2004). Trust and mistrust of online health sites. In Proceedings of the Conference on Human Factors in Computing Systems (pp. 663-670). New York: ACM Press.

Trevino, L., Lengel, R., \& Daft, R. (1987). Media symbolism, media richness, and media choice in organizations: A symbolic interactionist perspective. Communication Research, 14, 553-574.
Walther, J.B. (1996). Computer-mediated communication: Impersonal, interpersonal and hyperpersonal interaction. Communication Research, 23, 3-43.

Whitty, M.T., \& Carville, S.E. (2008). Would I lie to you? Self-serving lies and other-oriented lies told across different media. Computers in Human Behavior, 24, 1021-1031.

Williams, S.S. (2001). Sexual lying among college students in close and casual relationships. Journal of Applied Social Psychology, 31(11), 2322-2338.

Zhou, L., Burgoon, J.K., Nunmaker, J.F., \& Twitchell, D. (2004). Automating linguistic-based cues for detecting deception in text-based asynchronous computer-mediated communication. Group Decision and Negotiation, 13, 81-106. 